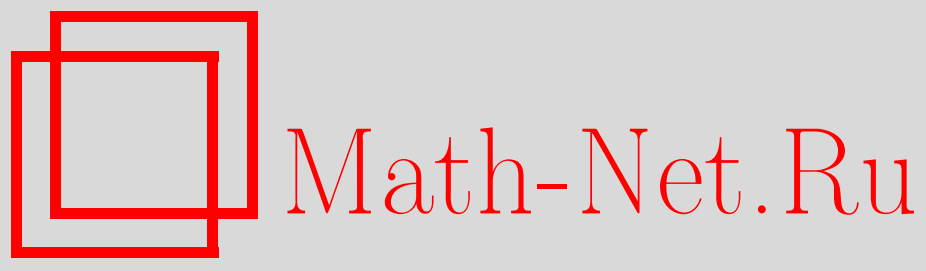

Е. А. Горин, Оценки инволюции разложимых элементов комплексной банаховой алгебры, Функи. анализ и его прил., 2005, том 39, выпуск 4, 14-31

DOI: https://doi.org/10.4213/faa82

Использование Общероссийского математического портала Math-Net.Ru подразумевает, что вы прочитали и согласны с пользовательским соглашением

http://www . mathnet.ru/rus/agreement

Параметры загрузки:

IP : 107.22 .136 .117

26 апреля 2023 г., 15:00:13

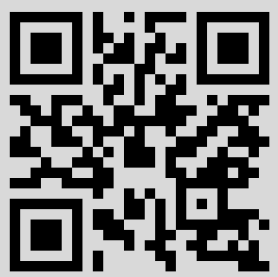


Функииональный анализ и его приложения

2005, т. 39, вып. 4, с. 14-31

УДК 517.9

\title{
Оценки инволюции разложимых элементов комплексной банаховой алгебры
}

\author{
(c) 2005. Е. А. Горин
}

\section{Введение}

В этой работе в основном речь идет о банаховых алгебрах $A$ с единицей $\mathbb{I}$ над полем $\mathbb{C}$ комплексных чисел. Когда необходимо уточнение, мы пишем $\mathbb{I}_{A}$ вместо II. С другой стороны, эпитеты «банахова» и «комплексная» зачастую опускаются. Поскольку нас будут интересовать метрические задачи, в определение алгебры включается предположение, что $\|\mathbb{I}\|=1$ и что $\|x y\| \leqslant\|x\| \cdot\|y\|$ для всех $x, y \in A$.

Как правило, мы будем придерживаться стандартной терминологии, принятой, например, в трактатах Н. Бурбаки или в монографии [1]. Кстати, в этой последней детально обсуждается часть из вводимых ниже (и подробнее обсуждающихся в §1) объектов и история их возникновения. Дополнительную информацию можно найти в статье [2], посвященной неравенствам Бернштейна, которые и здесь играют заметную роль. Классический подход к неравенствам Бернштейна и их обобщениям отражен во многих публикациях. Особенно я хотел бы выделить недавно вышедшее (на русском языке) собрание сочинений Н. И. Ахиезера [3] и последнюю монографию Б. Я. Левина [4] (русский текст которой, к сожалению, не опубликован).

$\mathbb{C}$-линейный функционал $\psi: A \rightarrow \mathbb{C}$ называется состоянием, если $\|\psi\|=$ $\psi(\mathbb{I})=1$. Например, в алгебре $A=C(X)$ всех непрерывных функций на метрическом компакте $X$ гомоморфизмам $\varphi: A \rightarrow \mathbb{C}$ отвечают (согласно теореме Рисса) точечные меры, а состояниям - произвольные вероятностные борелевские меры.

Очевидно, что множество $\operatorname{St}(A)$ всех состояний составляет $\sigma\left(A^{*}, A\right)$-компактное выпуклое подмножество сопряженного пространства $A^{*}$. В отличие от множества гомоморфизмов, которое в случае некоммутативной алгебры может оказаться пустым, множество состояний всегда весьма обильно.

Выпуклый компакт

$$
V(a) \stackrel{\text { def }}{=}\{\psi(a) \mid \psi \in \operatorname{St}(A)\}
$$

называется числовым образом элемента $а$. Радиус наименьшего диска с центром в точке 0 , содержащего $V(a)$, называется числовым радиусом элемента $a$ и обозначается через $v(a)$. Ясно, что $v(a) \leqslant\|a\|$. С другой стороны, известно, что $\|a\| \leqslant e \cdot v(a)$ (оценка точная).

Спектр элемента $a \in A$ мы обозначаем через $\operatorname{Spec}(a)$ или, если необходимо уточнение, $\operatorname{Spec}_{A}(a)$. Для спектрального радиуса используется обозначение $|a|$, и мы надеемся, что это не будет приводить к недоразумениям. Числовой образ 
содержит выпуклую оболочку спектра, но, вообще говоря, существенно шире этого множества (даже в классе двумерных коммутативных алгебр с единицей).

Элемент $a \in A$ называется эрмитовым, если $V(a) \subset \mathbb{R}$, где $\mathbb{R}$ - поле вещественных чисел. Множество всех эрмитовых элементов алгебры $A$ обозначается через $H(A)$. Это множество является замкнутым $\mathbb{R}$-линейным подпространством в $A$.

Так как $\operatorname{Spec}(a) \subset \mathbb{R}$ для всех $a \in H(A)$, то множество эрмитовых элементов одномерно для всех алгебр типа алгебры аналитических функций в диске. Заметим, что оно одномерно и для алгебры абсолютно сходящихся рядов Фурье. В случае $C^{*}$-алгебр эрмитовость совпадает с обычной самосопряженностью. Всегда $\|a\|=|a|$ для каждого $a \in H(A)$.

Элемент $x \in A$ называется разложимым, если он допускает представление $x=a+i b$, в котором $a$ и $b$ эрмитовы. Такое представление, если оно существует, единственно, так что эрмитовы компоненты $a, b$ разложимого элемента $x$ определены однозначно.

Совокупность всех разложимых элементов алгебры $A$ обозначается через $H_{\mathbb{C}}(A)$. Ясно, что $H_{\mathbb{C}}(A)$ - замкнутое $\mathbb{C}$-линейное подпространство в $A$, более того, $H_{\mathbb{C}}(A)$ - банахова алгебра Ли относительно коммутатора. Заметим, что если в (ассоциативной) банаховой алгебре все элементы разложимы, то, согласно теореме Виддава-Пальмера (см., например, [1]), эта алгебра имеет естественную $C^{*}$-структуру. Поэтому ясно, что замкнутость пространства $H_{\mathbb{C}}(A)$ относительно исходного умножения - большая редкость.

На алгебре $H_{\mathbb{C}}(A)$ определена естественная инволющия

$$
a+i b=x \rightarrow x^{*}=a-i b .
$$

Для разложимых элементов указанная выше оценка уточняется: $\|x\| \leqslant 2 v(x)$ (и здесь оценка точная). Так как $v(x)=v\left(x^{*}\right)$, то отсюда следует, что для разложимых элементов $\left\|x^{*}\right\| \leqslant 2\|x\|$.

Один из основных результатов данной работы состоит в том, что последнюю оценку можно уточнить: существует такая константа $\gamma<2$, что $\left\|x^{*}\right\| \leqslant \gamma\|x\|$. Наилучшее значение этой константы определяется равенством

$$
\gamma=2 \sup \left|f^{\prime}(0)\right|
$$

где верхняя грань берется по всем таким (вещественным нечетным) целым функциям $f$ экспоненциального типа $\leqslant 1$, для которых на вещественной оси

$$
\left|t^{n+1}\left(t^{-1} d / d t\right)^{n}(f(t) / t)\right| \leqslant 1 \quad \text { при всех } n=0,1,2, \ldots .
$$

Фактически изучается следующая более общая проблема. Будем рассматривать только такие разложимые $x \in A$, для которых $\left\|x^{*}\right\|=2$ и $v(x)=\tau$. Тогда $1 \leqslant \tau \leqslant 2$ и $\|x\| \geqslant \tau$. При фиксированном $\tau$ естественно спросить о такой наибольшей константе $c(\tau)$, что в этой ситуации непременно $\|x\| \geqslant c(\tau)$.

Ясно, что $\gamma=2 / \inf c(\tau)$. Оказывается, что

$$
c(1)=\max _{t>0}\left\{\frac{\sin t}{t}-\cos t\right\}=1,0631 \ldots
$$

и что функция $c(\tau)$ непрерывна и монотонно убывает при $\tau<\tau_{0}$. Если $\tau \geqslant \tau_{0}$, то $c(\tau)=\tau$. Конечно, $\gamma=2 / \tau_{0}$.

Небольшой список результатов настоящей статьи опубликован в [5]. 
Я хотел бы отметить, что в дальнейшем тексте можно обнаружить целый ряд естественных, но не решенных аналитических задач.

ЗАмечАниЕ. Аксиома выбора порой применяется к категориям, не являющимся множествами, и нам хотелось бы этого избежать. По смыслу нашей задачи в ней речь идет о паре элементов алгебры. Поэтому в качестве исходного материала мы можем рассматривать только алгебры с числом образующих $\leqslant 2$. Вместе с тем для каждого натурального $n$ существует такое множество $\mathfrak{M}(n)$ банаховых алгебр, что каждая банахова алгебра с числом образующих $\leqslant n$ изометрически изоморфна алгебре из $\mathfrak{M}(n)$. Действительно, пусть $\operatorname{Ass}_{\mathbb{C}}(n)$ - свободная ассоциативная алгебра с $n$ образующими над полем $\mathbb{C}$. Элементами этой алгебры служат «ассоциативные, но не коммутативные полиномы от $n$ переменных с комплексными коэффициентами». Алгебры вида $\operatorname{Ass}_{\mathbb{C}}(n) / I$, где $I$ - двусторонний идеал, составляют множество, и в качестве $\mathfrak{M}(n)$ можно взять конкретное множество пополнений (например, по Хаусдорфу) таких факторалгебр. В дальнейшем мы будем все это иметь в виду.

\section{§1. Предварительные сведения}

В этом параграфе мы изложим в основном хорошо известные факты. Для удобства ссылок некоторые из них оформляются в виде лемм.

Если $f$ - непрерывная вещественная выпуклая функция на полуоси $t \geqslant 0$, причем $f(0)=0$, то $f(t) / t$ не убывает и поэтому имеет предел при $t \rightarrow 0$.

Пусть $A$ - комплексное банахово пространство, $a, b \in A$. Тогда из выпуклости нормы и теоремы Хана-Банаха вытекает, что

$$
\lim _{t \rightarrow 0}(\|b+t a\|-\|b\|) / t=\sup \operatorname{Re} \psi(a),
$$

где верхняя грань берется по всем функционалам $\psi$, для которых $\|\psi\|=1$ и $\psi(b)=\|b\|$. Заметим, что двустороннего предела (при $t \rightarrow 0)$, вообще говоря, нет.

В применении к банаховым алгебрам получается, что для каждого элемента $x \in A$ и $t \geqslant 0$

$$
\|\mathbb{I}+t x\|=1+r(x) t+\varepsilon(t) t,
$$

где $\varepsilon(t) \geqslant 0$ - неубывающая функция, $\varepsilon(t) \rightarrow 0$ при $t \rightarrow 0$ и

$$
r(x)=\sup \{\operatorname{Re} \psi(x) \mid \psi \in \operatorname{St}(A)\} .
$$

Число $r(x)$ называется числовой абсииссой элемента $x$. Ясно, что $r(\lambda x)=\lambda r(x)$ при $\lambda \geqslant 0$ и $r(x+y) \leqslant r(x)+r(y)$.

Из формулы (1) получается, что элемент a тогда и только тогда эрмитов $($ т. е. $V(a) \subset \mathbb{R})$, когда $\|\mathbb{I}+i t a\|=1+o(t)$ в вещественной окрестности точки $t=0$.

Пусть $\omega=\omega(t)$ - такая неотрицательная функция на полуоси $t>0$, что $\omega(s+t) \leqslant \omega(s) \cdot \omega(t)$ при всех $s, t>0$.

ЛЕмма 1. Предположим, что $\sup _{t>0} \omega(t)^{1 / t}<\infty$. Тогда

$$
\varlimsup_{t \rightarrow \infty} \omega(t)^{1 / t} \leqslant \inf \omega(t)^{1 / t} \quad u \quad \varliminf_{t \rightarrow 0} \omega(t)^{1 / t} \geqslant \sup \omega(t)^{1 / t} .
$$

В частности, предель при $t \rightarrow \infty$ ири $t \rightarrow 0$ существуют.

Дискретный вариант леммы 1 называется леммой Фекете и тесно связан с формулой Гельфанда для спектрального радиуса. В этом случае условия на 
верхнюю грань не требуется, а второе утверждение теряет смысл. Доказательство обоих утверждений этой леммы не требует ничего, кроме стандартной процедуры «деления с остатком». Поэтому правую полуось можно заменить положительной частью всюду плотной подгруппы вещественной оси. С другой стороны, хотя дополнительные условия (вроде $\omega(t) \leqslant e^{c t}$ ) можно варьировать, совсем освободиться от них нельзя (примеры получаются из разрывных решений уравнения Коши-Абеля).

Из условия (1) и леммы 1 вытекает, что для эрмитова элемента $a$ при $t \rightarrow 0$, $t \in \mathbb{R}$, имеет место соотношение $\left\|e^{i t a}\right\|=1+o(t)$. Отсюда (и из леммы 1 ) легко следует, что элемент а тогда и только тогда эрмитов, когда $\left\|e^{i t a}\right\|=1$ при всех $t \in \mathbb{R}$ (довольно часто это свойство используется в качестве определения).

Из леммы 1 вытекает следующий аналог формулы (1):

$$
\log \left\|e^{t x}\right\|=r(x) t-\varepsilon(t) t,
$$

где $\varepsilon(t) \geqslant 0$ и $\varepsilon(t) \rightarrow 0$ при $t \rightarrow 0$. В частности, числовая абсцисса - это наименьшая из таких констант $r$, что $\left\|e^{t x}\right\| \leqslant e^{r t}$ при всех $t \geqslant 0$. Аналогично, числовой радиус $v(x)$ - это наименьшая из таких констант $v$, что

$$
\left\|e^{\lambda x}\right\| \leqslant e^{v|\lambda|} \quad \text { при всех } \lambda \in \mathbb{C} \text {. }
$$

Хотя $V(x)$ целиком определяется двумерным сечением алгебры (проходящим через $x$ и $\mathbb{I}$ ), при всех натуральных $n$ имеют место следующие неравенства:

$$
\left\|x^{n}\right\| \leqslant \frac{n ! e^{n}}{n^{n}} v(x)^{n} .
$$

Эти неравенства вытекают из (3) и формулы Коши. Сопоставляя неравенства (4) с формулой Стирлинга в форме

$$
n !=(n / e)^{n} \sqrt{2 \pi n} e^{\theta /(12 n)}, \quad \text { где } 0<\theta=\theta(n)<1,
$$

при $n \geqslant 1$ получаем (грубое, но прозрачное) неравенство

$$
\left\|x^{n}\right\| \leqslant 3 n^{1 / 2} v(x)^{n}
$$

$\left(\right.$ так как $\left.\sqrt{2 \pi} e^{1 / 12}=2,724 \ldots<3\right)$.

Если $a_{1}, a_{2} \in H(A)$ и $a_{1}+i a_{2}=0$, то $\psi\left(a_{1}\right)=\psi\left(a_{2}\right)=0$ для каждого $\psi \in$ $\operatorname{St}(A)$. Поэтому $a_{1}=a_{2}=0$. Следовательно, эрмитовы компоненты $a=\operatorname{Re} x$, $b=\operatorname{Im} x$ разложимого элемента $x=a+i b$ определены однозначно.

Так как $v(\operatorname{Re} x) \leqslant v(x)$, то $H_{\mathbb{C}}(A)$ - замкнутое подпространство в $A$. Кроме того, $i[a, b] \in H(A)$, если $a, b \in H(A)$, т. е. $H_{\mathbb{C}}(A)$ - банахова алгебра Ли. Действительно, пусть $t \in \mathbb{R}$ и $\psi \in \operatorname{St}(A)$. Положим

$$
\psi_{t}(x) \stackrel{\text { def }}{=} \psi\left(e^{i t a} x e^{-i t a}\right) .
$$

Легко видеть, что $\psi_{t} \in \mathrm{St}(A)$. Следовательно, $\psi_{t}(b) \in \mathbb{R}$, и остается взять производную $\partial \psi_{t}(b) / \partial t$ при $t=0$. Заметим, что элемент $a^{2}$ при эрмитовом $a$ может оказаться неразложимым.

Пусть $A-$ ассоциативное кольцо с единицей, и пусть $\varnothing \neq S \subset A$. Положим

$$
Z(S) \stackrel{\text { def }}{=}\{y \in A \mid x y=y x \text { для всех } x \in S\} .
$$

Следующая лемма - стандартный алгебраический факт: 
Лемма 2. Пусть $B=Z(Z(S))$. Тогда $B-$ подкольцо в $A$ и каждый элемент из $B$, обратимый в $A$, обратим и в $B$. Если элементы из $S$ коммутируют, то В коммутативно. Если $A$ - банахова алгебра, то $B$ - ее замкнутая подалгебра.

Доказательство имеется, например, в [6, гл. 11].

Поэтому если $A$ - банахова алгебра и $x \in A$, то $\operatorname{Spec}(x) \subset V(x)$ и, в частности, $|x| \leqslant v(x)$. Действительно, пусть $S=\{x\}$. Тогда $B-$ коммутативная банахова алгебра и, кроме того, $\operatorname{Spec}_{A}(x)=\operatorname{Spec}_{B}(x)$. В соответствии с основными принципами теории Гельфанда $\operatorname{Spec}_{B}(x)$ составляют числа вида $\varphi(x)$, где $\varphi-$ комплексный гомоморфизм алгебры $B$, так что $\|\varphi\|=\varphi(\mathbb{I})=1$. Поэтому, продолжая $\varphi$ с сохранением нормы на $A$, мы получим состояние.

В дальнейшем мы пишем $Z(x)$ вместо $Z(\{x\})$.

Хорошо известно, что $\|a\|=|a|$, если $a \in H(A)$. Это равенство фактически эквивалентно классическому неравенству С. Н. Бернштейна для производной целой функции экспоненциального типа, ограниченной на вещественной оси.

В случае эрмитовых элементов стандартное функциональное исчисление допускает естественное расширение за пределы аналитических функций.

Обозначим через $\operatorname{Sym}(\mathbb{R})$ алгебру символов, состоящую из всех комплексных функций на $\mathbb{R}$, локально совпадающих с преобразованиями Фурье-Стилтьеса мер конечной полной вариации. Если $q \in \operatorname{Sym}(\mathbb{R})$ и $a \in H(A)$, то по определению $q(a)=\int_{\mathbb{R}} e^{i t a} \nu(d t)$, где $\nu-($ какая-нибудь) мера, для которой $q(\lambda)=\hat{\nu}(\lambda)$ в некоторой вещественной окрестности компакта $\operatorname{Spec}(a)$. Сравнительно легко проверить, что $\operatorname{Spec}(q(a))=\{q(\lambda) \mid \lambda \in \operatorname{Spec}(a)\}$. Символ называется универсальным, если в описанной ситуации $\|q(a)\|=|q(a)|$. Это понятие, введенное в [7], детально обсуждается в [8] в контексте локально компактных абелевых групп. Имеются критерии универсальности в терминах положительно определенных функций, в ряде случаев допускающие простую проверку. В частности, если $q$ - полином первой степени (с комплексными коэффициентами), то $q$ и $e^{q}-$ универсальные символы.

Следующее неравенство уточняет оценку $\|x\| \leqslant e \cdot v(x)$ в случае разложимых элементов и является прямым обобщением известного неравенства Бергера (для $C^{*}$-алгебр):

Лемма 3. Если $x \in H_{\mathbb{C}}(A), m o\|x\| \leqslant 2 v(x)$.

Действительно,

$$
\|x\| \leqslant\|\operatorname{Re} x\|+\|\operatorname{Im} x\|=|\operatorname{Re} x|+|\operatorname{Im} x| \leqslant 2 v(x) .
$$

Разложимый элемент $x=a+i b$ будем называть нормальным, если $[a, b]=0$.

ЛЕмма 4. Если элемент $x$ нормален, то $V(x)$ совпадает с выпуклой оболочкой спектра этого элемента. В частности, $v(x)=|x|$.

Действительно, без ограничения общности можно предположить, что алгебра $A$ коммутативна. Достаточно установить, что $V(x)$ лежит в полуплоскости $\operatorname{Re} \lambda \leqslant 0$, если в этой полуплоскости лежит $\operatorname{Spec}(x)$. Пусть $t>0$ и $\varphi \in \operatorname{Hom}(A, \mathbb{C})$ (т. е. $\varphi-$ комплексный гомоморфизм). Пусть $a=\operatorname{Re} x$. Тогда $0<\varphi\left(e^{t a}\right)=e^{t \varphi(a)} \leqslant 1$. Поэтому $\left\|e^{t x}\right\|=\left\|e^{t a}\right\|=\left|e^{t a}\right| \leqslant 1$, т. е. $r(x) \leqslant 0$.

$\mathrm{B}$ частности, согласно леммам 3 и $4,\|x\| \leqslant 2|x|$ для нормальных элементов $x$, и эта оценка точна (ниже мы приведем пример). Вместе с тем вытекающая 
из равенства $V(x)=\overline{V\left(x^{*}\right)}$ и леммы 3 оценка $\left\|x^{*}\right\| \leqslant 2\|x\|$ точной не является, и установить это - одна из целей данной работы.

Классическое пространство Бернштейна - это пространство $\mathbf{B}_{\tau}$ всех целых функций $f$ экспоненциального типа не выше $\tau$, ограниченных на вещественной оси. Относительно поточечных операций и sup-нормы по $\mathbb{R}$ это пространство является банаховым. В комплексной плоскости $|f(\lambda)| \leqslant\|f\| e^{\tau|\operatorname{Im} \lambda|}$. Отсюда следует, что $\mathbf{B}_{\tau}$ составляют в точности те непрерывные ограниченные функции на оси, преобразование Фурье которых сосредоточено на отрезке $[-\tau, \tau]$. Это позволяет конструировать содержательные аналоги пространств $\mathbf{B}_{\tau}$ во всех $\mathbb{R}^{n}$ (и даже на локально компактных абелевых группах). Нам потребуется один из простейших примеров такого сорта.

В дальнейшем через $Q$ обозначается евклидов единичный шар в стандартном пространстве $\mathbb{R}^{2}$. Далее, при $\tau>0$ через $\mathbf{B}_{\tau}(Q)$ обозначается снабженное sup-нормой по плоскости пространство ограниченных непрерывных функций, преобразование Фурье которых сосредоточено на $\tau Q$. Эти функции продолжаются до целых в $\mathbb{C}^{2}$ с оценкой, аналогичной указанной (разумеется, теперь | $\operatorname{Im} \lambda \mid$ - евклидова норма).

Нам будет удобно ввести в $\mathbb{R}^{2}$ комплексную координату $z=\lambda_{1}+i \lambda_{2}$ и соответствующий оператор $D=\partial / \partial \lambda_{1}-i \partial / \partial \lambda_{2}$. Формально сопряженный оператор обозначается через $D^{*}$, так что, в частности, $D z=2, D^{*} z=0$ и $\Delta=D D^{*}-$ оператор Лапласа.

Определим неотрицательное $r$ равенством $r^{2}=z \bar{z}$. Если $g(t)-$ четная гладкая функция на вещественной оси, то $g(r)$ - сферически симметричная функция на $\mathbb{R}^{2}$. Ясно, что $D g(r)=\bar{z} g^{\prime}(r) / r$. Вообще, при неотрицательных $k$ и $l$

$$
D^{k} \bar{z}^{l} g(r)=\bar{z}^{k+l}\left(r^{-1} d / d r\right)^{k} g(r) .
$$

Вращения в $\mathbb{R}^{2}$ реализуются в форме $z \rightarrow \zeta z$, где $|\zeta|=1$. Обозначим через $R_{\zeta}$ индуцированный оператор в $\mathbf{B}_{\tau}(Q)$. Тогда $R_{\zeta}-$ изометрия и, кроме того, $D R_{\zeta}=\zeta R_{\zeta} D$. Положим

$$
P \stackrel{\text { def }}{=} \frac{1}{2 \pi i} \int_{|\zeta|=1} R_{\zeta} d \zeta
$$

Если $u \in \mathbf{B}_{\tau}(Q)$, причем

$$
u(z, \bar{z})=\sum_{p, q \geqslant 0} c_{p, q} z^{p} \bar{z}^{q},
$$

TO

$$
(P u)(z, \bar{z})=\bar{z} \sum_{p \geqslant 0} c_{p, p+1} r^{2 p}=\bar{z} g(r),
$$

где $g$ - четная функция из $\mathbf{B}_{\tau}$, причем $\sup _{\mathbb{R}}|t g(t)| \leqslant\|u\|$. Поэтому (нечетная) функция $f(t)=t g(t)$ также принадлежит $\mathbf{B}_{\tau}$ и $\|f\| \leqslant\|u\|$.

Ясно, что $\left(D^{*} u\right)(0,0)=2 f^{\prime}(0)$. По неравенству Бернштейна $\left|f^{\prime}(0)\right| \leqslant \tau\|f\|$, и среди нечетных $f$ равенство достигается в точности для функций, кратных $\sin \tau t$.

В пространстве $\mathbf{B}_{\tau}(Q)$ оператор $D$ является нормальным (с сопряженным $\left.D^{*}\right)$ и $\operatorname{Spec}(D)=\tau Q$. Поэтому $v(D)=|D|=\tau$. С другой стороны, если $u(z, \bar{z})=$ $(z \sin \tau r) / r$, то $\|u\|=1$ и $(D u)(0,0)=2 \tau$, так что $\|D\|=2 \tau$. Аналогично, $\left\|D^{*}\right\|=$ $2 \tau$, так как $\left(D^{*} v\right)(0,0)=2$, где $v(z, \bar{z})=(\bar{z} \sin \tau r) / r$. Других таких функций в 
$\mathbf{B}_{\tau}(Q)$ нет [7], и это довольно просто вытекает из описания экстремалей для неравенства Бернштейна в одномерном случае.

Существует несколько способов доказать, что $\left\|D^{2}\right\|=8 \tau^{2} / \pi$, но уже вычисление $\left\|D^{3}\right\|$ приводит к полным эллиптическим интегралам. При $n \geqslant 1$ грубую оценку сверху для $\left\|D^{n}\right\|$ дает формула (5):

$$
\left\|D^{n}\right\| \leqslant 3 n^{1 / 2} \tau^{n}
$$

а грубую оценку снизу - соотношение

$$
\left\|D^{n}\right\|^{2} \geqslant\left\|\Delta^{n}\right\|=4^{n}(n !)^{2} \tau^{2 n} /(2 n) ! \sim(\pi n)^{1 / 2} \tau^{2 n} .
$$

\section{§2. Предварительные конструкции и оценки}

$\mathrm{B}$ дальнейшем для вещественных $\xi, \eta \in \mathbb{R}$ будет удобно вместо $\max \{\xi, \eta\}$ писать $\xi \oplus \eta$.

Пусть $A$ и $B$ - банаховы алгебры. Через $A \oplus B$ мы обозначаем прямую сумму алгебр $A$ и $B$ с нормой $\|a \oplus b\|=\|a\| \oplus\|b\|$. Исходные алгебры естественно погружаются в $A \oplus B$ в качестве идеалов. Алгебра $A \oplus B$ тогда и только тогда коммутативна, когда коммутативны обе алгебры $A$ и $B$.

Лемма 5. $V(a \oplus b)$ - выпуклая оболочка множества $V(a) \cup V(b)$. B частности, $v(a \oplus b)=v(a) \oplus v(b)$.

ДОКАЗАТЕЛЬСТвО. «Тонкость» состоит в том, что $a, b$ и $a \oplus b-$ элементы различных алгебр.

Если $\psi \in \operatorname{St}(A)$, то $x \oplus y \rightarrow \psi(x)$ - состояние алгебры $A \oplus B$. Поэтому $V(a) \subset V(a \oplus b)$, и остается проверить, что $r(a \oplus b) \leqslant r(a) \oplus r(b)$. Но если $r(a)=r(b)=0$, то это очевидно (так как $A \oplus B$ снабжается «нормой Кратана»), а общий случай сводится к этому ${ }^{1)}$.

Пусть $A_{1}, A_{2}, \ldots$ - последовательность банаховых алгебр. Обозначим через $\Sigma$ банахову алгебру всех ограниченных (по норме) последовательностей $x=$ $\left(x_{1}, x_{2}, \ldots\right), x_{k} \in A_{k}$, с покоординатными операциями и нормой $\sup _{k}\left\|x_{k}\right\|$.

Рассмотрим факторалгебру $\Sigma / \Sigma_{0}$, где $\Sigma_{0}$ - идеал последовательностей из $\Sigma$, для которых $x_{k} \rightarrow 0$ при $k \rightarrow \infty$.

ЛЕмма 6. Предположим, что элемент $x \in \Sigma / \Sigma_{0}$ порождается последовательностъю $\left\{x_{k}\right\}$. Тогда $\|x\|=\varlimsup_{k \rightarrow \infty}\left\|x_{k}\right\|, r(x)=\varlimsup_{k \rightarrow \infty} r\left(x_{k}\right) u v(x)=$ $\varlimsup_{k \rightarrow \infty} v\left(x_{k}\right)$.

ДокАЗАТЕЛЬСтво. Первое утверждение тривиально. Докажем второе утверждение.

Предположим, что $\varlimsup_{k \rightarrow \infty} r\left(x_{k}\right)<0$. Заменяя последовательность эквивалентной, мы можем считать, что $r\left(x_{k}\right)<0$ при всех $k$. По формуле $(2)$ тогда $\log \left\|e^{t x_{k}}\right\|<0$ при каждом $t>0$. Следовательно, $\log \left\|e^{t x}\right\| \leqslant 0$, так что $r(x) \leqslant 0$.

Обратно, пусть $r(x)<0$. Тогда $\left\|\mathbb{I}+t_{0} x\right\|<1$ при некотором $t_{0}>0$. Обозначим через $\mathbb{I}_{k}$ единицу алгебры $A_{k}$. Заменяя последовательность эквивалентной, можно считать, что $\left\|\mathbb{1}_{k}+t_{0} x_{k}\right\|<1$ при всех $k$. По формуле $(1)$ тогда $t_{0} r\left(x_{k}\right)<0$.

Последнее утверждение можно доказать, используя формулу (3), однако легко свести дело к числовым абсциссам, и мы ограничимся этим замечанием.

1) Напомним, что $r(a)$ - это числовая абсцисса элемента $а$ (а не спектральный радиус, который обозначается через $|a|)$. 
Заметим, кроме того, что $|x|$ может оказаться больше соответствующего верхнего предела, и это аналогично тому, что последовательность нильпотентов может сходиться по норме к элементу с положительным спектральным радиусом, однако в данном случае пример указать проще: достаточно в качестве $A_{n}$ взять алгебру всех операторов гильбертова пространства размерности $n$ с отмеченным ортогональным базисом $\left\{e_{k}\right\}$ и рассмотреть в $A_{n}$ жорданову клетку

$$
e_{n} \rightarrow e_{n-1} \rightarrow \cdots \rightarrow e_{2} \rightarrow e_{1} \rightarrow 0
$$

максимальной размерности.

Пусть $X$ - банахово пространство и $B=B(X)$ - алгебра всех ограниченных операторов $S: X \rightarrow X$, снабженная стандартной операторной нормой.

Пусть $T \in B(X)$, причем $|T|<\|T\|$. Фиксируем такую константу $c$, что $|T|<c$, и введем в $X$ новую норму по формуле

$$
\llbracket x \rrbracket=\sup _{k \geqslant 0} c^{-k}\left\|T^{k} x\right\| \text {. }
$$

Легко видеть, что это определение корректно, что новая норма эквивалентна исходной и что $\|x\| \leqslant \llbracket x \rrbracket$ для всех $x \in X$. Хотя новая норма зависит от $T$ и $c$, мы не будем снабжать ее дополнительными индексами.

Легко проверить, что $\llbracket S x \rrbracket \leqslant\|S\| \cdot \square x \rrbracket$ для всех $x \in X$, если $S \in Z(T)$. Следовательно, в таком случае $\llbracket S \square \leqslant\|S\|$, где $] S \square$ - операторная норма, порожденная новой нормой на $X$.

Пусть $Y$ - еще одно банахово пространство. При замене исходной нормы в $X$ на новую и сохранении нормы в $Y$ нормы операторов $X \rightarrow Y$ не возрастают. В частности, это относится к нормам линейных функционалов на $X$.

Лемма 7. (а) Если $S \in Z(T) u\|S x\|=\|x\|$ для всех $x \in X$, то и $\llbracket S x \rrbracket=\llbracket x \rrbracket$ для всех $x \in X$.

(b) $\llbracket T \rrbracket \leqslant c$.

(c) $E$ сли $c \leqslant\|T\|$, mо $\square T \square=c$.

(d) Если оператор $T$ нормален в исходной норме, то он нормален и в новой.

ДокАзАтельство. Утверждения (a) и (b) тривиальны. Докажем утверждение (с). Если $c=\|T\|$, то, как легко видеть, $\|x\|=\rrbracket x \rrbracket$ при всех $x \in X$ и доказывать нечего. Поэтому будем считать, что $c<\|T\|$. Но в этом случае найдется такой вектор $x_{0} \in X$, что $\left\|x_{0}\right\|<c^{-1}\left\|T x_{0}\right\|$. Легко проверить, что тогда $\llbracket x_{0} \rrbracket=c^{-1} \llbracket T x_{0} \rrbracket$, так что $\llbracket T \rrbracket \geqslant c$ (а обратное неравенство содержится в п. (b)). Наконец, пусть $T$ - оператор, нормальный в исходной норме. Тогда $\operatorname{Re} T$ эрмитов, так что $\left\|e^{i t \operatorname{Re} T}\right\|=1$ при всех вещественных $t$. Согласно утверждению (a), это свойство сохранится и в новой норме, т. е. Rе $T$ остается эрмитовым. Лемма доказана.

ЗАмЕчаниЕ. Утверждение (d) обобщается: если $S$ нормален и $S \in Z(T)$, то $S$ нормален и в новой норме. К доказательству надо добавить только одно: установить, что $\operatorname{Re} S \in Z(T)$. Но этот факт является весьма частным случаем обобщенной (см., например, [9]) теоремы Путнама-Фугледе: оператор, коммутирующий с нормальным, коммутирует и с сопряженным.

Рассмотрим последовательность $A_{1} \supset A_{2} \supset A_{3} \supset \cdots$ банаховых алгебр с общей единицей. Для простоты норма элемента из $A_{n+1}$ предполагается не меньшей, чем норма этого элемента в $A_{n}$. Алгебра $\mathbf{A} \stackrel{\text { def }}{=} \bigcap_{1}^{\infty} A_{n}$ снабжается 
стандартной топологией проективного предела. В этой топологии $\mathbf{A}$ - полная метризуемая локально выпуклая алгебра.

Для каждого $\mathbf{x} \in \mathbf{A}$ имеет смысл экспонента $e^{\mathbf{x}}$, причем $\mathbf{u}(t)=e^{t \mathbf{x}}$ является единственным решением задачи Коши $\dot{\mathbf{u}}=\mathbf{x u}(t), \mathbf{u}(0)=\mathbb{I}$.

Линейный непрерывный оператор $\mathbf{D}: \mathbf{A} \rightarrow \mathbf{A}$ называется дифференцированием, если $\mathbf{D} \mathbf{x y}=(\mathbf{D} \mathbf{x}) \mathbf{y}+\mathbf{x}(\mathbf{D y})$ для всех $\mathbf{x}, \mathbf{y} \in \mathbf{A}$. Заметим, что формула Лейбница (с учетом порядка сомножителей) сохраняется.

Приведенное ниже рассуждение аналогично тем, которые обычно используются в теории возмущений ${ }^{1)}$.

ЛЕмма 8. Для каждого непрерьвного дифферениирования $\mathbf{D}: \mathbf{A} \rightarrow \mathbf{A}$, каждого $\mathbf{x} \in \mathbf{A}$, кажсдого $t \in \mathbb{R} u$ кажндого натурального $n$

$$
\mathbf{D}^{n} e^{t \mathbf{x}}=\sum_{1}^{n}\left(\begin{array}{l}
n \\
k
\end{array}\right) \int_{0}^{t} e^{(t-\tau) \mathbf{x}}\left(\mathbf{D}^{k} \mathbf{x}\right)\left(\mathbf{D}^{n-k} e^{\tau \mathbf{x}}\right) d \tau
$$

ДокАЗАТЕЛЬСтво. Положим $\mathbf{v}=\mathbf{D}^{n} \mathbf{u}$, где $\mathbf{u}=e^{t \mathbf{x}}$. Тогда $\mathbf{v}(0)=0$ и

$$
\dot{\mathbf{v}}=\mathbf{x} \cdot \mathbf{v}+\sum_{1}^{n}\left(\begin{array}{l}
n \\
k
\end{array}\right)\left(\mathbf{D}^{k} \mathbf{x}\right)\left(\mathbf{D}^{n-k} \mathbf{u}\right) \text {. }
$$

Так как $\mathbf{u}$ обратим, мы можем искать $\mathbf{v}$ в виде $\mathbf{v}=\mathbf{u z}$. Для $\dot{\mathbf{z}}$ получается явное представление, и остается учесть, что $\mathbf{z}(0)=0$.

СЛЕДСтвиЕ. Если дополнителъно $\mathbf{D}^{2} \mathbf{x}=0$, mо

$$
\mathbf{D}^{n} e^{t \mathbf{x}}=n \int_{0}^{t} e^{(t-\tau) \mathbf{x}}(\mathbf{D} \mathbf{x})\left(\mathbf{D}^{n-1} e^{\tau \mathbf{x}}\right) d \tau .
$$

Разумеется, при $n=1$ формула (7) - частный случай леммы 8 , так что дополнительного условия $\mathbf{D}^{2} \mathbf{x}=0$ не требуется.

Следующий пример наиболее важен для дальнейшего. Пусть $A$ - банахова алгебра, $m$ - натуральное число и $\mathbf{A}=\operatorname{Hol}\left(\mathbb{C}^{m}, A\right)$ - алгебра всех целых $A$-значных функций от $\lambda=\left(\lambda_{1}, \ldots, \lambda_{m}\right) \in \mathbb{C}^{m}$. Мы снабжаем алгебру $\mathbf{A}$ поточечными операциями и топологией равномерной сходимости на компактах. Ясно, что к алгебре $\mathbf{A}$ применима лемма 8. Вместе с тем сужение $\mathbf{A} \rightarrow \mathbf{A} \mid \mathbb{R}^{m}$ - алгебраический изоморфизм, и мъ можем рассматривать $\mathbf{A}$ как алгебру $A$-значных функиий на $\mathbb{R}^{m}$.

Пусть $\zeta_{1}, \ldots, \zeta_{m} \in \mathbb{C}$ и

$$
\mathbf{D} \stackrel{\text { def }}{=} \zeta_{1} \frac{\partial}{\partial \lambda_{1}}+\cdots+\zeta_{m} \frac{\partial}{\partial \lambda_{m}} .
$$

Тогда $\mathbf{D}$ - непрерывное дифференцирование алгебры $\mathbf{A}$. Пусть $a_{1}, \ldots, a_{m} \in A$ и $\mathbf{x}=\lambda_{1} a_{1}+\cdots+\lambda_{m} a_{m}$. Очевидно, что $\mathbf{x} \in \mathbf{A}$ и $\mathbf{D}^{2} \mathbf{x}=0$.

Теорема 1. Пусть $a_{1}, \ldots, a_{m} \in H(A)$. Тогда при вещественньх $\lambda_{k}$, комплексных $\zeta_{k}$ и натуральных $n$ имеет место неравенство

$$
\left\|\left(\zeta_{1} \frac{\partial}{\partial \lambda_{1}}+\cdots+\zeta_{m} \frac{\partial}{\partial \lambda_{m}}\right)^{n} e^{i\left(\lambda_{1} a_{1}+\cdots+\lambda_{m} a_{m}\right)}\right\| \leqslant\left\|\zeta_{1} a_{1}+\cdots+\zeta_{m} a_{m}\right\|^{n} .
$$

1) На такое рассуждение (для $n=1$ ), существенно более короткое и прозрачное, чем мое первоначальное (использовавшее формулу Далецкого-Троттера), в свое время указал мне В. Я. Лин. 
ДокАЗАТЕЛЬСтво. Из тождества (7) по индукции легко получается, что при всех $t>0$ имеет место неравенство $\left\|\mathbf{D}^{n} e^{i t \mathbf{x}}\right\| \leqslant t^{n}\|\mathbf{D} \mathbf{x}\|^{n}$, в котором остается взять $t=1$.

\section{§3. Схема изменения функции $c(\tau)$}

Обозначим через $T$ множество (треугольник) всех таких точек $(\tau, \omega) \in \mathbb{R}^{2}$, что

$$
1 \leqslant \tau \leqslant \omega \leqslant 2
$$

Ясно, что относительно покоординатной операции $\xi \oplus \eta$ это множество составляет компактную абелеву полугруппу.

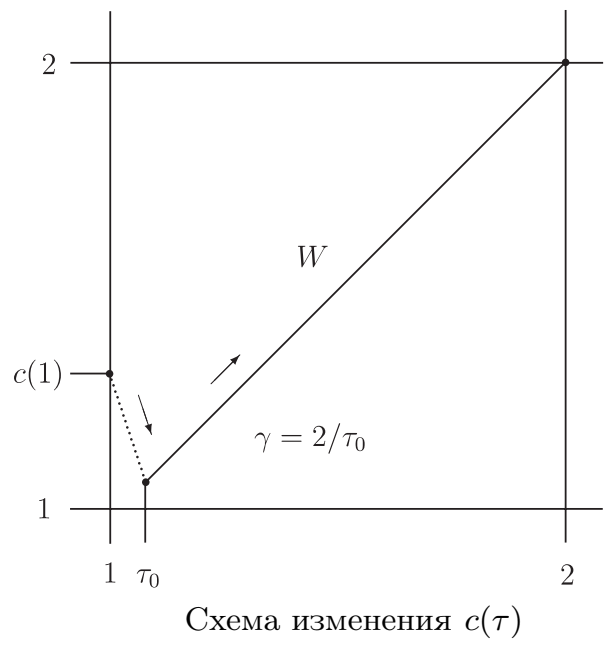

Обозначим через $W$ совокупность всех таких точек $(\tau, \omega) \in \mathbb{R}^{2}$, координаты которых допускают реализацию $\tau=v(x), \omega=\|x\|$, где $x$ - разложимый элемент (хотя бы одной) банаховой алгебры, для которого $\left\|x^{*}\right\|=2$.

Если $0 \leqslant \alpha \leqslant \beta \leqslant 2 \alpha$, то найдется такой нормальный элемент $x$, что $v(x)=\alpha$ и $\|x\|=\left\|x^{*}\right\|=\beta$. Действительно, можно считать, что $\beta=2$. Если при этом $\alpha=1$, то в качестве $x$ можно взять оператор $D$ в пространстве $\mathbf{B}_{1}(Q)$ (с исходной нормой, см. конец $\S 1)$. При $\alpha>1$ вместе с этим элементом $x$ достаточно рассмотреть элемент $\alpha$ в одномерной алгебре $\mathbb{C}$ и применить к $x \oplus \alpha$ лемму 5 .

Сопоставляя этот факт с леммой 6 , легко убедиться, что $W$ - замкнутый идеал в $T$.

ЗАмЕчАниЕ. Если точка $\xi \in W$ реализуется нормальным элементом и $\eta \in T$, то из сказанного выше вытекает, что $\xi \oplus \eta$ также реализуется нормальным элементом. На самом деле, как будет доказано в следующем параграфе, каждая точка из $W$ реализуется нормальным элементом, однако для доказательства этого факта нам придется использовать утверждение, содержащееся в предыдущей фразе.

Основная характеристика, с которой мы будем иметь дело, - это функция $c(\tau)$ на отрезке $1 \leqslant \tau \leqslant 2$, которая определяется равенством

$$
c(\tau)=\inf \{\omega \mid(\tau, \omega) \in W\} .
$$


Так как $W$ - замкнутое подмножество в $T$, то нижняя грань достигается. Далее, легко убедиться, что если $c\left(\tau_{0}\right)=\tau_{0}$ при некотором $\tau_{0} \geqslant 1$, то $c(\tau)=\tau$ при всех $\tau \geqslant \tau_{0}$.

В дальнейшем через $\tau_{0}$ мы будем обозначать наименьшее из всех таких $\tau$, для которых $c(\tau)=\tau$.

Лемма 9. На интервале $\left(1, \tau_{0}\right)$ функиия $c(\tau)$ не возрастает.

ДоказАТЕЛЬСтво. Пусть $1<\tau_{1}<\tau_{0}$. Тогда $\tau_{1}<\omega_{1}=c\left(\tau_{1}\right)$. Отрезок, соединяющий точки $\left(\tau_{1}, \omega_{1}\right)$ и $\left(\omega_{1}, \omega_{1}\right)$, содержится в $W$. Поэтому $c(\tau) \leqslant \omega_{1}$ всюду на отрезке $\left(\tau_{1}, \omega_{1}\right)$.

ЗАмечАниЕ. Последняя лемма приобретет смысл, когда мы докажем, что $\tau_{0}>1$, т. е. что $c(1)>1$.

Так как множество $W$ замкнуто, легко убедиться, что функция $c(\tau)$ непрерывна справа. На самом деле она непрерывна и $c(\tau)<c(1)$ при $1<\tau \leqslant \tau_{0}$, но для доказательства этих утверждений потребуются дополнительные средства. Приведенная выше схема дает некоторое представление об изменении $c(\tau)$. На этой схеме масштабы в левом нижнем углу примерно в 10 раз больше, чем в правом верхнем). Некоторые элементы этой схемы будут обоснованы только в $\S 5$.

\section{§4. Функциональная модель}

Приводимые ниже утверждения естественно объединяются в функциональный критерий включения $(\tau, \omega) \in W$. Однако если вставить в формулировку сопутствующие обстоятельства, то она удлиняется и теряет прозрачность. Поэтому мы разделяем ее на несколько отдельных утверждений. Как и выше, мы используем формальные переменные $z$ и $\bar{z}$, так что $u=u(z, \bar{z})$.

Теорема 2. Пусть $x \neq 0-$ разложимый элемент алгебры $A$. Тогда существует такая функиия $u \in \mathbf{B}_{\tau}(Q)$, где $\tau=v(x)$, что $\left\|D^{k} u\right\| \leqslant\|x\|^{k}$ при всех $k \geqslant 0 u\left(D^{*} u\right)(0,0)=\left\|x^{*}\right\|$.

ДокАЗАТЕЛЬСтво. Пусть $x=a_{1}+i a_{2}$, где $a_{1}=\operatorname{Re} x$. Рассмотрим функцию $F(\lambda)=e^{i\left(\lambda_{1} a_{1}-\lambda_{2} a_{2}\right)}$ от $\lambda=\left(\lambda_{1}, \lambda_{2}\right) \in \mathbb{C}^{2}$ со значениями в $A$. Пусть $\lambda=\xi+i \eta$, так что, например, $\xi_{1}=\operatorname{Re} \lambda_{1}$.

Из теоремы 1 вытекает, что $\left\|D^{k} F(\xi)\right\| \leqslant\|x\|^{k}$.

Далее, $i\left(\lambda_{1} a_{1}-\lambda_{2} a_{2}\right)=a+i b$, где $a=-\eta_{1} a_{1}+\eta_{2} a_{2}, b=\xi_{1} a_{1}-\xi_{2} a_{2}$ и оба элемента эрмитовы. Ясно (см. начало $\S 1)$, что $\left\|e^{a+i b}\right\| \leqslant e^{r(a+i b)} \leqslant e^{v(a)}$. Пусть $\psi \in \operatorname{St}(A)$. Тогда $|\psi(a)| \leqslant\left(\eta_{1}^{2}+\eta_{2}^{2}\right)^{1 / 2}\left(\psi\left(a_{1}\right)^{2}+\psi\left(a_{2}\right)^{2}\right)^{1 / 2}$, так что $v(a) \leqslant|\eta| v(x)$. Иначе говоря, $\|F(\lambda)\| \leqslant \tau|\operatorname{Im} \lambda|$. Наконец, ясно, что $\left(D^{*} F\right)(0,0)=i x^{*}$.

Таким образом, в качестве искомого $u$ можно взять $\chi \circ F$, где $\chi \in A^{*},\|\chi\|=1$ и $\chi\left(i x^{*}\right)=\left\|x^{*}\right\|$. Теорема доказана.

ЗАмечАнИЕ. Если к $u$ применить операцию $P$, введенную в $\S 1$, то получится функция с аналогичными свойствами вида $\bar{z} f(r) / r$, где $f$ - нечетная вещественная функция из $\mathbf{B}_{\tau}$.

TeOpema 3. Пусть $(\tau, \omega) \in T$, и пусть $u \in \mathbf{B}_{\tau}(Q)$, причем $\left(D^{*} u\right)(0,0)=2$ $($ или $\geqslant 2) u\left\|D^{k} u\right\| \leqslant \omega^{k}$ при всех иельх $k \geqslant 0$. Тогда найдется такой нормальный элемент $x$, что $v(x)=\tau,\|x\|=\omega u\left\|x^{*}\right\|=2$, m.е. $(\tau, \omega)$ принадлежит $W$ и реализуется нормальным элементом. 
ДокАЗАтЕльство. Не ограничивая общности, можно считать, что $1 \leqslant \tau<$ $\omega<2$. Снабдим пространство $\mathbf{B}_{\tau}(Q)$ нормой $\llbracket v \rrbracket$, введенной перед леммой 7,

$$
\square v \square=\sup _{k \geqslant 0} \omega^{-k}\left\|D^{k} v\right\| \text {. }
$$

Тогда $v(D)=\tau$ и $[D]=\omega$.

На алгебре операторов пространства $\mathbf{B}_{\tau}(Q)$ с исходной нормой рассмотрим функционал $\chi(L) \stackrel{\text { def }}{=}(L u)(0,0)$. Ясно, что $\|\chi\|=1$. Поэтому $\square \chi \square \leqslant 1$. Так как $\chi\left(D^{*}\right) \geqslant 2$, то $\lambda \stackrel{\text { def }}{=} \llbracket D^{*} \llbracket \geqslant 2$.

Итак, мы построили такой нормальный элемент $x$, что $v(x)=\tau,\|x\|=\omega$ и $\left\|x^{*}\right\|=\lambda \geqslant 2$. Теперь воспользуемся замечанием из начала $\S 3$ и предшествующими ему рассуждениями. Векторы $(2 \tau / \lambda, 2 \omega / \lambda, 2)$ и $(\tau, \omega, \omega)$ из $\mathbb{R}^{3}$ оба реализуются нормальными элементами, и потому такую же реализацию имеет и их $\oplus$-сумма (покоординатный максимум), т. е. $(\tau, \omega, 2)$. Теорема доказана.

СлЕДСТВИЕ 1. Все точки из $W$ имеют нормальную реализацию.

СлЕДСтвиЕ 2. Пусть $1 \leqslant \tau \leqslant \omega \leqslant 2$. Включение $(\tau, \omega) \in W$ имеет место тогда и только тогда, когда существует такая нечетная вещественная бункиия $f \in \mathbf{B}_{\tau}$, что $f^{\prime}(0)=1$ u $\left|t^{n+1}\left(t^{-1} d / d t\right)^{n}(f(t) / t)\right| \leqslant \omega^{n}$ при всех цельх $n \geqslant 0$ и всех вещественных $t$.

\section{§5. Основные свойства функции $c(\tau)$}

Последнее утверждение предыдущего параграфа естественно приводит к рассмотрению последовательности функций $\left\{f_{n}\right\}, n \geqslant 0$, которая формируется следующим образом. Пусть $f$ - нечетная вещественная функция из $\mathbf{B}_{\tau}$. Положим $f_{0}=f$ и

$$
f_{n}(t)=(-1)^{n} t^{n+1}\left(t^{-1} d / d t\right)^{n}(f(t) / t)
$$

при $n \geqslant 1$. Выбор знака таков, что при $f(t)=\sin t$ в качестве $f_{n}$ получаются классические функции Риккати-Бесселя $s_{n}(t)=(\pi t / 2)^{1 / 2} J_{n+1 / 2}(t)$. Положим $c_{n}=\left\|s_{n}\right\|(\sup$-норма по $\mathbb{R})$.

Следующая теорема (из которой легко вытекает, что $\gamma<2$ ) - один из основных результатов данной работы.

Теорема 4. Величина с(1) представляется (приведенной во введении) бормулой

$$
c(1)=\max _{t>0}\left\{\frac{\sin t}{t}-\cos t\right\}=1,06310 \ldots
$$

ДокАЗАтЕльство. Для краткости будем писать $c$ вместо $c(1)$. Существует такой разложимый элемент $x$, что $\|x\|=c, v(x)=1,\left\|x^{*}\right\|=2$. Согласно следствию 2 из теоремы 3, найдется такая нечетная вещественная функция $f \in$ $\mathbf{B}_{1}$, что $\left\|f_{n}\right\| \leqslant c^{n}$ (так что, в частности, $\left.\|f\| \leqslant 1\right)$ и $f^{\prime}(0)=1$. Поэтому описание экстремалей в одномерном неравенстве Бернштейна влечет за собой равенство $f(t)=\sin t$. Следовательно, $c=\sup _{n \geqslant 1} c_{n}^{1 / n}$. Чтобы завершить доказательство теоремы 4, теперь достаточно установить, что справедлива следующая

ЛЕмма 10. Если $n \geqslant 2$, mо $c_{n}<c_{1}^{n}$. 
ДокАЗАТЕльство ${ }^{1)}$. Пусть $t_{n}-$ минимальная положительная критическая точка функции $s_{n}$. Хорошо известно, что $\left|s_{n}(t)\right|<c_{n}=s_{n}\left(t_{n}\right)$, если $n \geqslant 1$ и $t \neq t_{n}$. Кроме того, модули значений в критических точках строго убывают при удалении от точки 0 и их предел равен 1.

Хотя функции $s_{n}$ элементарные, их вид быстро усложняется. Тем не менее простейшие вычислительные средства позволяют установить, что $t_{1}=2,74370 \ldots$, что $c_{1}=1,06310 \ldots$, а также что $c_{2}<c_{1}^{2}$ и $c_{3}<c_{1}^{3}$.

Оценка (4) при $\tau=1$ и $n \geqslant 1$ приводит к оценке $c_{n} \leqslant\left\|D^{n}\right\| \leqslant 3 n^{1 / 2}$, из которой легко вытекает, что неравенство $c_{n}<c_{1}^{n}$ достаточно проверить при $n \leqslant m=50$. Эту проверку уже можно «поручить» достаточно развитому калькулятору .... Если воспользоваться связями функций Бесселя с полиномами Лежандра, то можно снизить $m$ до 20. Наконец, можно свести конкретные вычисления до указанного выше минимума $(m=3)$, и в заключение мы кратко опишем, как это сделать.

Пусть $Y_{\nu}$ - функция Неймана. Известно (см., например, [10, с. 491]), что для $\nu \geqslant 1 / 2$ функция

$$
\left(t^{2}-\nu^{2}\right)^{1 / 2}\left(J_{\nu}(t)^{2}+Y_{\nu}(t)^{2}\right)
$$

при $t \geqslant \nu$ не убывает и стремится к $2 / \pi$. Следовательно, при $t>\nu$

$$
\left((\pi t / 2)^{1 / 2} J_{\nu}(t)\right)^{4}<t^{2} /\left(t^{2}-\nu^{2}\right) .
$$

Если $\nu>4$, то первая стационарная положительная точка для $J_{\nu}(t)$ лежит правее $\nu^{1 / 2}(\nu+3)^{1 / 2}$ (теорема Шафхейтлина [10, с. 536]). Тем более это верно для произведения на $(\pi t / 2)^{1 / 2}$. Кроме того, модуль этого произведения в первой стационарной точке достигает глобального максимума [10, с. 537]. Так как $t^{2} /\left(t^{2}-\nu^{2}\right)$ при $t>\nu$ убывает, то получается, что указанное произведение не превосходит $(1+\nu / 3)^{1 / 4}$ и потому $s_{n}^{4}(t)^{4}<(2 n+7) / 6$ при $n \geqslant 4$. Легко убедиться, что при таких $n$ правая часть в последнем неравенстве меньше $c_{1}^{4 n}$, так что $c_{n}<c_{1}^{n}$. Лемма доказана и тем самым завершено доказательство теоремы 4.

Используя замену $\xi \rightarrow \xi / \tau$, критерий включения $(\tau, \omega) \in W$ можно переформулировать в терминах класса $\mathbf{B}_{1}(Q)$ или класса $\mathbf{B}_{1}$ функций одной переменной экспоненциального типа $\leqslant 1$. В доказательстве следующей теоремы мы используем тот факт, что указанное включение имеет место тогда и только тогда, когда существует такая функция $u \in \mathbf{B}_{1}(Q)$, что $\left(D^{*} u\right)(0,0)=2$ и

$$
\left\|D^{k} u\right\| \leqslant \omega^{k} / \tau^{k-1} \quad \text { при всех целых } k \geqslant 0 .
$$

ТеоРема 5. Функиия $c(\tau)$ непрерывна.

ДоКАЗАТЕЛЬСТво. Мы будем использовать обозначения (и факты) из 3. Достаточно рассмотреть промежуток $\left(1, \tau_{0}\right]$ и проверить непрерывность слева. Пусть $1<\sigma \leqslant \tau_{0}$. Точка $\sigma$ остается фиксированной.

Пусть $u_{0}$ - экстремальная функция из $\mathbf{B}_{1}(Q)$, отвечающая точке $\tau=1$ (так что $\left.u_{0}(z, \bar{z})=(\bar{z} \sin r) / r\right)$, и $u_{1}$ - экстремальная функция, отвечающая точке $\sigma$ (так что для этой функции выполняются неравенства (9) с $\tau=\sigma$ и $\left.\omega=\omega_{1}=c(\sigma)\right)$. При $0<\varepsilon<1$ положим $u_{\varepsilon}=\varepsilon u_{0}+(1-\varepsilon) u_{1}$. Ясно, что

1) Такие вещи могли быть известны лет 200 назад. Вместе с тем, поскольку речь идет о сравнении значений в переходных зонах, конкретные вычисления становятся почти неизбежными. 
$u_{\varepsilon} \in \mathbf{B}_{1}(Q)$ и $\left(D^{*} u_{\varepsilon}\right)(0,0)=2$. Фиксируем $\delta>0$ и займемся оценками норм $\left\|D^{k} u_{\varepsilon}\right\|$.

При $k=0$ имеем

$$
\left\|u_{\varepsilon}\right\| \leqslant \tau_{\varepsilon} \stackrel{\text { def }}{=} \varepsilon+(1-\varepsilon) \sigma .
$$

Ясно, что $1<\tau_{\varepsilon}<\sigma$ и что $\tau_{\varepsilon} \rightarrow \sigma$ при $\varepsilon \rightarrow 0$.

При всех $k \geqslant 1$ имеет место неравенство $\left\|D^{k} u_{\varepsilon}\right\| \leqslant 3 k^{1 / 2} \tau_{\varepsilon}$. Следовательно, если $k_{0}$ достаточно велико, то при $k>k_{0}$

$$
\left\|D^{k} u_{\varepsilon}\right\| \leqslant(1+\delta)^{k} \omega_{1}^{k} / \tau_{\varepsilon}^{k-1},
$$

так как $\tau_{\varepsilon}<\sigma \leqslant \omega_{1}$. Если мы проверим, что такое же неравенство выполняется при всех $k \leqslant k_{0}$ и всех достаточно малых $\varepsilon>0$, то получится, что

$$
\lim _{\varepsilon \rightarrow 0} c\left(\tau_{\varepsilon}\right) \leqslant(1+\delta) \omega_{1}
$$

и теорема будет доказана.

При $k=0$ неравенство выполняется при всех $\varepsilon$. Рассмотрим для определенности случай $k=2$. Имеем

$$
\left\|D^{2} u_{\varepsilon}\right\| \leqslant \varepsilon c^{2}(1)+(1-\varepsilon) \omega_{1}^{2} / \sigma \leqslant(1+\delta)^{2} \omega_{1}^{2} / \tau_{\varepsilon},
$$

если $\varepsilon>0$ достаточно мало (так как $\tau_{\varepsilon} \rightarrow \sigma$ при $\varepsilon \rightarrow 0$; разумеется, $(1+\delta)^{2}$ написано для единообразия). При другом $k$, быть может, придется уменьшить $\varepsilon$, однако эту процедуру достаточно проделать не более $k_{0}$ раз. Доказательство закончено.

ЗАмЕчАниЕ. Пусть $\left\{c_{n}\right\}$ - последовательность глобальных максимумов функций Риккати-Бесселя. Используя известные асимптотики максимумов бесселевых функций (см., в частности, [11, 9.5.24]), нетрудно убедиться, что при $n \rightarrow \infty$ имеет место эквивалентность $c_{n} \sim a n^{1 / 6}$. Это наводит на мысль, что последовательность $\left\{c_{n}\right\}$ возрастающая, а последовательность $\left\{c_{n}^{1 / n}\right\}$ убывающая. Я не умею этого доказывать. При небольших $n$ оба утверждения подтверждаются вычислениями. В пользу второго утверждения говорит и лемма 10. Более того, если $n>2$, mо $c_{n}^{1 / n}<c_{2}^{1 / 2}$. Чтобы в этом убедиться, достаточно повторить доказательство леммы 10 (однако теоремы Шафхейтлина приводят к цели только при $n>5$, а остающиеся случаи проверяются при помощи вычислений).

Мы воспользуемся неравенством $c_{n}^{1 / n}<c_{2}^{1 / 2}$ при доказательстве следующей теоремы (оно сокращает путь, хотя можно было бы обойтись и без него).

ТеОрема 6. Неравенство $c(1+\varepsilon)<c(1)$ имеет место при всех достаточно малых $\varepsilon>0$.

Доказательство. Пусть $\varepsilon>0$. Построим последовательность $\left\{f_{n}\right\}$, начиная не $\mathrm{c} \sin t$, а с $f_{0}(t)=(\sin t)(\cos \varepsilon t)$. Достаточно убедиться, что при малых $\varepsilon>0$ и всех $k \geqslant 0$ будут выполняться неравенства

$$
b_{k} \stackrel{\text { def }}{=} \sup _{\mathbb{R}}\left|f_{k}(t)\right| \leqslant b^{k}, \quad \text { где } b<c(1) .
$$

Ясно, что $2 f_{0}(t)=\sin (1+\varepsilon) t+\sin (1-\varepsilon) t$. Пусть $c_{2}^{1 / 2}<d<c_{1}$. Имея в виду отмеченный выше факт, легко усмотреть, что $b_{k} \leqslant((1+\varepsilon) d)^{k}$ при $k \geqslant 2$. 
Следовательно, если $0<\varepsilon<\delta$, где $(1+\delta) d<c_{1}$, то неравенства $(10)$ с $b=(1+\delta) d$ будут выполняться при $k \geqslant 2$. Поэтому решающим оказывается случай $k=1$.

Здесь удобно писать $f_{1}(t, \varepsilon)$, явно указывая зависимость от параметра $\varepsilon$.

Пусть $t_{1}>0$ - точка первого максимума функции $s_{1}(t)=f_{1}(t, 0)$. Мы знаем, что $t_{1} \in(2,3)$. Пусть $\Delta_{1}-$ интервал длины $2\left(3-t_{1}\right)$ с центром в точке $t_{1}$. На множестве $\mathbb{R} \backslash \Delta_{1}$ функция $s_{1}(t)$ строго меньше, чем $c_{1}$, и это свойство сохранится при всех достаточно малых $\varepsilon>0$. Наконец,

$$
\left.\frac{\partial^{2} f_{1}}{\partial \varepsilon^{2}}\right|_{\varepsilon=0}=-t^{2} s_{1}(t)-2 t \sin t<0,
$$

если $t \in \Delta_{1}$. Поэтому $\sup _{t}\left|f_{1}(t, \varepsilon)\right|<c_{1}$ при всех достаточно малых $\varepsilon>0$, и теорема доказана.

Можно предположить, что на промежутке $\left[1, \tau_{0}\right)$ функция $c(\tau)$ строго убывает. Увы, ни доказать это в общем объеме, ни опровергнуть я не умею. Следующее рассуждение показывает, что в некоторой правой полуокрестности точки 1 строгое убывание есть.

Если $\left\{f_{k}\right\}$ - стандартная последовательность (которая начинается с ограниченной на $\mathbb{R}$ функции $f_{0}$ экспоненциального типа), то по индукции легко получается, что

$$
f_{k}(t)=(-1)^{k} f_{0}^{(k)}(t)+O\left(t^{-1}\right),
$$

причем $O$-член допускает равномерную оценку по $f_{0}$ с ограниченными в совокупности экспоненциальными типами и верхними гранями модуля на $\mathbb{R}$.

При $1<\tau<\tau_{0}$ отметим (какую-нибудь) «экстремальную функцию» $f_{0, \tau}$. Выберем (какую-нибудь) последовательность $\tau \rightarrow 1$ (не будем снабжать $\tau$ индексами). Из этой последовательности (по теореме Витали) можно извлечь подпоследовательность, равномерно сходящуюся на компактах. Предположим, что мы перешли к подпоследовательности. Тогда имеет место сходимость $f_{0, \tau} \rightarrow f_{0}$. Легко видеть, что предельная функция - экстремаль, отвечающая значению $\tau=1$, так что $f_{0}(t)=\sin t$. Таким образом, если $\tau \rightarrow 1$, то $f_{0, \tau}(z)$ равномерно на компактах сходится к $\sin z$. Из теоремы Вейерштрасса вытекает, что в том же смысле имеет место сходимость $f_{k, \tau} \rightarrow s_{k}$ при каждом $k \geqslant 0$.

Сопоставляя этот факт сходимости с оценками (11), мы получим, что для каждого фиксированного $k$ при $\tau$, близких к 1 , график функции $f_{0, \tau}$ в своей существенной части почти не отличается от графика функции $s_{k}$. Например, для $f_{1, \tau}$ первая положительная стационарная точка будет точкой глобального максимума, а вторая - точкой глобального минимума. Действительно, если бы, например, глобальный максимум распадался при $\tau \rightarrow 1$, то у $s_{1}^{\prime}$ возник бы положительный нуль высокой кратности (а такого нет).

Поэтому доказательство теоремы 6 в том, что касается набора $\left\{s_{k}\right\}$ с $k \leqslant k_{0}$, распространяется на $f_{k, \tau}$, если $\tau$ достаточно близко к 1 . Вместе с тем оценки функций с большими $k$ носили общий характер. Таким образом, при $\tau$, близких к 1 , теорема 6 переносится с функции $\sin t$ на все $f_{0, \tau}$, т. е. при таких $\tau$ функция $c(\tau)$ строго убывает.

Для константы $\gamma$, помимо принципиальной оценки $\gamma<2$, мы пока не имеем ничего, кроме неравенства $\geqslant 2 / c(1)$ (чуть лучшая оценка снизу на этом пути возможна, но связана с не очень приятными выкладками). Чтобы скольконибудь конкретно оценить $\gamma$ сверху, мы в заключительном параграфе оценим 
снизу $\tau_{0}=c\left(\tau_{0}\right)$. Заметим, что следствие 2 теоремы 3 приводит к сформулированной во введении экстремальной проблеме в $\mathbf{B}_{1}$, дающей точное значение константы $\gamma$. Однако я не знаю, как выглядит экстремаль.

\section{§6. Оценка снизу, связанная с $c(\tau)$}

Значение $c(\tau)$ - это нижняя грань таких $\omega$, для которых существует нечетная вещественная функция $f=f_{0} \in \mathbf{B}_{1}$, удовлетворяющая условиям $f^{\prime}(0)=1$ и $\left\|f_{k}\right\| \leqslant \omega^{k} / \tau^{k-1}$ при всех целых $k \geqslant 0$, причем эта нижняя грань реализуется. При $\tau=\tau_{0}$ имеет место равенство $\omega=\tau\left(\right.$ здесь $\left.\omega=c\left(\tau_{0}\right)\right)$. Если $f-$ экстремаль, отвечающая этой ситуации, то $\left\|f_{k}\right\| \leqslant \tau$. Уже неравенство $\|f\| \leqslant 1$ вместе с равенством $f^{\prime}(0)=1$ позволяет (как мы увидим) дать некоторую оценку снизу для $f_{1}$ на промежутке $(\pi / 2, \pi)$. Этот промежуток включает первую стационарную точку функции $s_{1}$, и, добавляя условие $\left\|f_{1}\right\| \leqslant \tau$, мы получим некоторую конструктивную оценку снизу для $\tau_{0}$ (и, стало быть, оценку сверху для $\left.\gamma=2 / \tau_{0}\right)$. Таким образом, как оценка сверху $\left(\tau_{0}<c(1)\right)$, так и оценка снизу основаны на весьма грубых приближениях. Поэтому финальное равенство $\gamma \approx 1,92$ с относительной погрешностью $2 \%$ можно расценивать как (относительную) удачу.

Мы начнем с простейшей интерполяционной формулы. Для краткости положим $\lambda_{n}=\pi / 2+\pi n$, где $n=0,1,2, \ldots$ Иначе говоря, $\Lambda \stackrel{\text { def }}{=}\left\{\lambda_{n}\right\}-$ последовательность всех положительных нулей функции $\cos t$. Пусть $f-$ нечетная функция из $\mathbf{B}_{1}$. Тогда равномерно на компактах имеет место представление

$$
\frac{f(z)}{z}=2 \cos z \sum_{n=0}^{\infty} \frac{(-1)^{n} f\left(\lambda_{n}\right)}{\lambda_{n}^{2}-z^{2}} .
$$

При $z=\lambda_{n}$ правая часть «по непрерывности» меняется на $f\left(\lambda_{n}\right) / \lambda_{n}$. Так как $f(t) / t$ принадлежит $L^{2}(\mathbb{R})$, то формула $(12)$ вытекает из (достаточно широко понимаемой) теоремы Пифагора.

Пусть $\nu$ - мера на оси $\mathbb{R}$, сосредоточенная на множестве $\Lambda$, причем $\nu\left(\left\{\lambda_{n}\right\}\right)=$ $2 \cdot(-1)^{n} / \lambda_{n}^{2}$. Тогда $\|\nu\|=1$ (норма меры - ее полная вариация). Представление (12) можно переписать в интегральной форме:

$$
\frac{f(t)}{t}=\int_{0}^{\infty} K_{0}(\lambda, t) f(\lambda) \nu(d \lambda), \quad \text { где } K_{0}(\lambda, t)=\frac{\lambda^{2} \cos t}{\lambda^{2}-t^{2}} .
$$

Заметим, что мы намеренно пишем интеграл так, как это принято в теории вероятностей. Для «независимой переменной» мы вместо $z$ снова употребляем $t$, поскольку не собираемся покидать вещественную ось.

Считая теперь, что $f=f_{0}$, из представления (13) мы получим представление

$$
f_{1}(t)=\int_{0}^{\infty} K_{1}(\lambda, t) f(\lambda) \nu(d \lambda), \quad \text { где } K_{1}(\lambda, t)=t \lambda^{2}\left(\frac{\sin t}{\lambda^{2}-t^{2}}-\frac{2 t \cos t}{\left(\lambda^{2}-t^{2}\right)^{2}}\right) .
$$

Нам потребуется несколько элементарных неравенств, которые для удобства ссылок мы оформим в виде лемм. Так как $\cos t<0$ в промежутке $(\pi / 2, \pi)$, то имеет место

ЛЕмма 11. Если $\pi<\alpha<\beta u \pi / 2<t<\pi$, mо $K_{1}(\alpha, t)>K_{1}(\beta, t)$. 
«Теоретическое» доказательство следующей леммы - довольно унылое занятие. Однако, поскольку речь идет об элементарном неравенстве с одной переменной, которое выполняется с некоторым запасом, можно использовать «достаточно продвинутый» калькулятор.

ЛЕмма 12. Если $\pi / 2<t<\pi$, то $K_{1}\left(\lambda_{1}, t\right)>K_{1}\left(\lambda_{0}, t\right)$.

Соединяя леммы 11 и 12 , мы получаем

СлЕДСТВИЕ. Если $\pi / 2<t<\pi$, mo

$$
\sup _{n} K_{1}\left(\lambda_{n}, t\right)=K_{1}\left(\lambda_{1}, t\right) \text {. }
$$

ЗАМЕчАНИЕ. При других значениях $t$ это, вообще говоря, неверно. Например, $\lim _{t \rightarrow 0} t^{-2} K_{1}(\lambda, t)=1-2 / \lambda^{2}$.

ЛЕмма 13. Если $0 \leqslant t \leqslant \pi$, то $K_{1}\left(\lambda_{n}, t\right) \geqslant 0$ при каждом $n \geqslant 0$.

ДокАзАтЕльство. Достаточно проверить, что в указанном промежутке $g(t) \geqslant 0$, где $g(t)=\left(\lambda_{0}^{2}-t^{2}\right) \sin t-2 t \cos t$, так что $g^{\prime}(t)=\left(\lambda_{0}^{2}-t^{2}-2\right) \cos t$. Уравнение $g^{\prime}(t)=0$ имеет 2 корня в интервале $(0, \pi)$, больший из которых есть $\pi / 2$, и в этой точке $g$ имеет минимум, равный 0 . Лемма доказана.

Обозначим через $\mu$ сосредоточенную на $\Lambda$ вероятностную меру, которая определяется равенствами $\mu\left(\left\{\lambda_{n}\right\}\right)=\left|\nu\left(\left\{\lambda_{n}\right\}\right)\right|$. Можно сказать, что $\mu(d \lambda)=$ $(\sin \lambda) \nu(d \lambda)$.

ТЕОРЕмА 7. Пусть $\tau>1, u$ пусть $f-$ такая нечетная вещественная функиия из $\mathbf{B}_{1}$, что $\|f\| \leqslant \tau u f^{\prime}(0)=1$. Пусть $f_{1}(t)=-(t d / d t)(f(t) / t)$. Тогда

$$
f_{1}(t) \leqslant \tau s_{1}(t), \quad \text { ecлu } 0 \leqslant t \leqslant \pi
$$

$u$

$$
f_{1}(t) \geqslant \tau s_{1}(t)-(\tau-1) K_{1}\left(\lambda_{1}, t\right), \quad \text { если } \pi / 2 \leqslant t \leqslant \pi .
$$

ДокАзАтельство. Дважды используя формулу (14) и лемму 13, получаем при $0 \leqslant t \leqslant \pi$

$$
f_{1}(t)=\int_{0}^{\infty} K_{1}(\lambda, t) f(\lambda) \nu(d \lambda) \leqslant \tau \int_{0}^{\infty} K_{1}(\lambda, t) \mu(d \lambda)=\tau s_{1}(t),
$$

и неравенство (16) установлено. Далее, если $\pi / 2 \leqslant t \leqslant \pi$, то

$$
\begin{aligned}
\tau s_{1}(t)-f_{1}(t) & =\sum_{n=0}^{\infty} K_{1}\left(\lambda_{n}, t\right)\left(\tau-(-1)^{n} f\left(\lambda_{n}\right)\right) \frac{2}{\lambda_{n}} \\
& \leqslant\left(\sup _{n} K_{1}\left(\lambda_{n}, t\right)\right) \sum_{n=0}^{\infty}\left(\tau-(-1)^{n} f\left(\lambda_{n}\right)\right) \frac{2}{\lambda_{n}} \\
& \left.=K_{1}\left(\lambda_{1}, t\right)\left(\tau-2 \sum_{n=0}^{\infty} \frac{(-1)^{n} f\left(\lambda_{n}\right)}{\lambda_{n}^{2}}\right) \quad \text { (по формуле }(15)\right) \\
& =K_{1}\left(\lambda_{1}, t\right)\left(\tau-f^{\prime}(0)\right)=(\tau-1) K_{1}\left(\lambda_{1}, t\right),
\end{aligned}
$$

и теорема доказана.

Применим теорему 7 в случае, когда $f$ - экстремаль, отвечающая точке $\tau_{0}$. Тогда в дополнение к неравенству (17) выполняется неравенство $\left\|f_{1}\right\| \leqslant \tau_{0}$. Поэтому в данном случае неравенство сохранится, если заменить $f_{1}(t)$ на $\tau_{0}$. 
Если произвести такую замену и в полученном неравенстве в качестве $t$ взять первую положительную стационарную точку $t_{1}$ функции $s_{1}(t)$, то получится, что

$$
\tau_{0} \geqslant K_{1}\left(\lambda_{1}, t_{1}\right) /\left(K_{1}\left(\lambda_{1}, t_{1}\right)-c(1)+1\right)=1,0212 \ldots .
$$

Среднее арифметическое правой части с $c(1)$ как раз и приводит к $\gamma \approx 1,92$.

\section{ЛитеРАТУРА}

1. Bonsall F. F., Duncan J. Complete Normed Algebras. Springer-Verlag, New YorkHeidelberg-Berlin, 1973.

2. Горин E. A. Неравенства Бернштейна с точки зрения теории операторов. Вестник Харьков. унив., сер. прикл. мат. и мех., 205, вып. 45, 77-105 (1980).

3. Ахиезер Н. И. Избранные труды по теории функций и математической физике. Т. 1, 2, Харьков, АКТА, 2001.

4. Levin B. Ya. Lectures on entire functions. Transl. Math. Monographs, Vol. 150, Amer. Math. Soc., Providence, RI, 1966.

5. Gorin E. A. Estimation of a partial involution in a Banach algebra. Russian J. Math. Phys., 5, № 1, 117-118 (1997).

6. Рудин У. Функциональный анализ. Мир, М., 1975.

7. Норвидас C. T. Об устойчивости дифференциальных операторов в пространствах целых функций. Докл. АН СССР, 291, вып. 3, 548-581 (1986).

8. Gorin E. A. Universal symbols on locally compact Abelian groups. Bull. Polish Acad. Sci., Math., 51, №2, 199-204 (2003).

9. Горин E. A. Обобщение одной теоремы Фугледе. Алгебра и анализ, 5, №4, 83-97 (1993).

10. Ватсон Г. Н. Теория бесселевых функций. ИЛ, М., 1949.

11. Абрамовии М., Стиган И. (ред.) Справочник по специальным функциям. Наука, M., 1979.

Московский педагогический государственный университет e-mail: evgeny.gorin@mtu-net.ru
Поступило в редакцию 28 июня 2005 г. 